\title{
Students' Understanding of Continuous Charge Distributions
}

\author{
Tharindu Jayasinghe and Homeyra Sadaghiani \\ Department of Physics and Astronomy, California State Polytechnic State University, 3801 W Temple Ave, Pomona, CA, 91768
}

\begin{abstract}
We have been investigating students' knowledge pertaining to continuous charge distributions in introductory calculus based physics courses at Cal Poly Pomona. An open-ended questionnaire was developed based on feedback from three faculty who often teach introductory calculus based electricity and magnetism courses to engineering and physics majors in order to probe common observed difficulties. A pilot study was conducted $(N=145)$ and, based upon the feedback we obtained from these results, a slightly revised version of the questionnaire with three additional questions was subsequently given to a second sample of students $(N=263)$ in the following quarter. Our data suggests that students struggle with concepts related to linear, surface, and volume charge distributions, as well as the mathematical formulation of infinitesimal charge representations of continuous charge distributions. We will share specific examples and discuss the implications that our findings have for more effective instruction.
\end{abstract}

\section{INTRODUCTION}

Students tend to have a better grasp of mechanics than electromagnetism. Research shows that students have difficulty transitioning from classical mechanics to electromagnetism due to the different methodological approaches used in each of these topics [1]. It has also been shown that students infrequently tie concepts from electrostatics with the description of macroscopic phenomena [2]. High school seniors rarely approach college with a coherent framework of ideas about electromagnetism [3]. Therefore, students often face challenges when taking courses on electromagnetism at the college level.

In most introductory physics courses, students first begin learning about discrete charge using Coulomb's law, and are then introduced to the concepts of continuous charge distributions in the latter parts of these courses. The transition from learning about discrete point charges to continuous charge distributions troubles many students. This is most likely due to the introduction of abstract concepts related to continuous charge that involve the use of the infinitesimal charge element $(d q)$ and mathematical difficulties related to the use of calculus in solving physics problems. Instructors teaching calculus based introductory physics courses typically expect students to use concepts of continuous charge distributions and infinitesimal charge elements to solve a variety of problems, including but not limited to, calculating the electric field and scalar potential of a given charge distribution and calculating the net charge possessed by a charged object. Given that the continuous charge distributions provide a more realistic description of charge in classical electrodynamics, students' robust understanding of these concepts is key to their success in more advanced courses. Our study focuses on understanding how students work with continuous charge distributions and how these students apply mathematics to solve these problems.

Some students struggle in introductory physics courses due to inadequate knowledge of calculus and the disconnect between mathematical formalism and physics. Students are expected to have calculus as a prerequisite or corequisite before enrolling in introductory calculus based physics courses.
Thus, instructors expect most students to be familiar with simple integration. However, it has been shown that instructors commonly overestimate the students' depth of understanding of mathematical concepts [4]. Studies have shown that a majority of the students do not have a satisfactory understanding of integration, even after taking calculus courses in college [5]. Even though most students are capable of evaluating integrals, they lack a conceptual understanding of the purpose of the integration [6]. It has been suggested that the Riemann-sum based conceptualization of integrals works best to help students understand integral expressions [7].

The disconnect between mathematical formalism and physics is apparent in the study of electromagnetism. Studies have shown that students, while capable of recognizing the need for an integral in the solution of a problem, often fail to set up the integral correctly [8]. This failure is attributed to the students' inability to understand the infinitesimal charge element commonly encountered in integrals used in electromagnetism [8]. These studies suggest that most students are unable to bridge their mathematical skills with physical concepts. This issue persists as students progress with their studies. A recent study has shown that students taking upper division courses in electrodynamics have difficulty visualizing the integrals as a sum [9], hence they are challenged in solving problems with continuous charge distributions [10]. Our Physics Education Research (PER) literature review resulted in very few focused studies describing how introductory physics students fare with the concepts of continuous charge, and thus more research needs to be done on this topic.

Motivated to enhance student success when they are first introduced to continuous charge distributions, we investigate student reasoning in lower division electromagnetism courses. The main purpose behind this study is to understand the major obstacles students face when they first encounter these topics. To achieve this, we first designed a 7-item pilot open-ended questionnaire to gage students' reasoning in electrostatic problems that involve continuous charge distributions and infinitesimal charge elements. We evaluated student answers and used the feedback from their responses to design an additional 3 questions in the second version of the 
questionnaire. In this paper, we will discuss the methodology in more detail, present example questions, analyze student responses, and discuss the implications these may have for the instructors of similar introductory electromagnetism courses.

\section{RESEARCH STUDY}

California State Polytechnic University Pomona (Cal Poly Pomona) is a Hispanic serving university and has a current enrollment of 9,700 science and engineering students with a majority $(57 \%)$ male population. The students who participated in this study were a mix of engineering, computer science and physics majors. These students were expected to have taken a calculus course covering the techniques of integration before enrolling in this calculus based physics course. Responses collected were anonymous and students were not given any course credit for taking part in the study. The pilot questionnaire was administered to 3 sections of the introductory calculus based electromagnetism class consisting of a total of 145 students. This class covers basic electrostatics, electrodynamics and circuits. The second revised questionnaire was administered in 4 sections of the introductory calculus based electromagnetism course which consisted of a total of 263 students.

\section{METHODS}

We developed a 7-item questionnaire based on feedback from three veteran physics faculty at Cal Poly Pomona. To gain insight into student ideas and probe possible sources for their difficulties, we designed the questionnaire in an openended format and asked students to explain their reasoning and thought process. The questionnaire tested students on the following topics: Uniform Charge Distributions (UCD), Continuous Charge Distributions (CCD) (Linear, Surface and Volume), Formulating the Infinitesimal Charge (FIC), the Change of Net charge $Q$ vs. Infinitesimal charge $d q$ (CNI). The questionnaire contained questions that tested the students on their understanding of physical concepts and mathematical skills (e.g., set-up integrals for a given physical quantity). Particular emphasis was given to gage students' understanding of the infinitesimal charge element and its use in integration. Students were also tested on dimensional analysis in order to test their understanding of linear, surface, and volume charge densities.

To better understand students' ideas that were expressed in some of their written responses in the pilot questionnaire, we produced a second version of the questionnaire by including three additional questions for a total of 10 questions (see $T A B L E I$ ). Minor revisions were also made to the wording of the questions in the pilot questionnaire. As an example of an additional question included in the second version of our questionnaire, we asked students to sketch a uniform and non-uniform surface charge distribution on an isolated insu- lating sphere. This was added to build upon the thought process of the previous question which asked students to define a uniform charge distribution, and to understand student visual mental models for uniformity or the lack thereof in charge distributions.

We find that a majority of the students struggle with concepts of continuous charge distributions and the use of calculus in solving problems involving continuous charge. We explore the results of our study in the next section while analyzing student responses to understand common difficulties and pitfalls encountered when working with electromagnetism.

\section{RESULTS}

The questions covered in our questionnaire broadly fall under the following topics: uniform charge distributions, continuous charge distributions and infinitesimal charge. Students were also asked how the total net charge $Q$ and infinitesimal charge $d q$ would vary when the charged objects were halved. We present our results and analysis of the questionnaire responses in the following four sub-sections. For the purposes of our analysis, we considered responses that were given partial credit to be incorrect because these responses did not completely arrive at the expected solution.

\section{A. Uniform Charge Distributions}

Most students had little difficulty when asked to define the term "uniform charge distribution" (60\% correct in the first version and $71 \%$ in the second version). Incorrect responses generally had to do with a misunderstanding of what uniformity entailed in the context of electrostatics. We expected students to understand that in a uniform charge distribution the total charge is equally distributed within or on the object.

To ensure that our results were not biased due to poor written communication skills in describing the concepts, and to help capture responses from those students who might have more precise mental models, students were also asked to sketch a uniform and a non-uniform surface charge distribution on two (insulating) spherical shells in the second version of the questionnaire. To our surprise only $36 \%$ of the 263 students answered this correctly. $30 \%$ of the student representations did not distinguish between the surface charge distributions of a shell from the volume charge distribution of a sphere. In many of the drawings, it seemed as if the charge existed within the spherical shell even when they were explicitly asked to sketch surface charge distributions. Based on these responses, we speculate that some of the issues with drawing a valid representation stem from the students' inability to visualize $3 \mathrm{D}$ objects and phenomena within a physical context.

Another noteworthy incorrect answer resulted from $25 \%$ of the students drawing a polarized object with a net zero charge by including an equal number of positive and negative charge 
TABLE I. Structure of the revised questionnaire, the number within the () indicates the number of questions in the pilot questionnaire.

\begin{tabular}{clcc}
\hline \hline Theme & \# of Questions & Topics & \% Correct \\
\hline UCD & $2(1)$ & Definition of uniform charge, Sketching a uniform charge distribution & 60 \\
CCD & $2(2)$ & Expressing charge densities in terms of the total charge, Units of charge densities & 44 \\
FIC & $4(2)$ & Expressing $d q$ in terms of charge densities, Units of $d q$, Integrals for charge, Purpose of integration & 25 \\
CNI & $2(2)$ & Change in net charge $Q$ vs. Infinitesimal charge $d q$ & 35 \\
\hline \hline
\end{tabular}

in their sketches. These students incorrectly thought that uniformity stemmed from an equal distribution of positive and negative charge, and did not realize that they were essentially drawing a null/neutral charge distribution.

\section{B. Continuous Charge Distributions}

Students were shown illustrations of three commonly encountered charge distributions: a sphere, disk and wire (see $F I G .1$ ) and were asked to express the appropriate charge density in terms of the net charge $Q$ possessed by each object. Over half of the students had difficulty attempting this problem. A third of the students were able to identify a relationship between the total charge and the charge density for the linear wire but were unable to replicate the same thought process for charge densities of higher dimensions. Roughly a quarter of these students were able to identify the correct relationship between the total charge and the charge density, but were unable to algebraically solve for the charge density. Students also thought that the linear charge density applied even to the disk and sphere, hinting at a conceptual misunderstanding of charge densities. Roughly a tenth of these students had trouble remembering the formulae needed to calculate the volume of the sphere and the surface area of the disk.

Students were also asked to carry out dimensional analysis for each of the charge densities encountered in the previous question. We found that students had trouble working with units and applying concepts of dimensional analysis to charge densities. Over a third of the students thought that the units of the charge density were the same for all the three different charge distributions, regardless of their dimensions. Furthermore, roughly $15 \%$ of the students wrote down units of mass instead of charge, suggesting that students were more familiar with mass densities.

\section{Formulating Infinitesimal Charge}

Students were guided through a problem asking them to solve for the total charge $Q$ of a given object. They were explicitly asked to use integration to arrive at their answer. Students found this problem especially challenging. Even though they were asked to express the infinitesimal charge $d q$ in terms of the appropriate charge density for each ob- ject (FIG. 1), the majority of the students did not effectively demonstrate their understanding of the concept of infinitesimal charge and the skills to use integration to solve such problems. Approximately $70 \%$ of the students did not seem to understand the terminology: infinitesimal charge, even when it was followed by the common algebraic notation $d q$. These students did not know how to proceed with defining $d q$ for each object and were unable to identify a proper relationship between $d q$ and the appropriate charge density. In the second version of the questionnaire, students were asked to express the units of the infinitesimal charge $d q$. Surprisingly, only $34 \%$ of the students were able to answer this correctly. Most students were not able to relate infinitesimal charge to the more common discrete point charge in order to answer this question. Common incorrect responses included inverse length units $\left(\mathrm{m}^{-1}\right)$ and units of energy $(J)$.

Students were then asked to write down general integrals to calculate the total charge possessed by each of the three objects. Although we believe that students were given adequate time to complete the questionnaire, over half of the students did not attempt this question, and the majority of the students who did try to solve this question struggled with connecting the mathematical concept of integration with the physics behind charge distributions. Only $17 \%$ of the students were able to answer this part correctly. However, one must consider that these questions were given to a number of course sections taught by different instructors who may or may not have covered the topics discussed in this paper in adequate detail.

\section{Change in $Q$ and $d q$}

In a two part question, students were asked to describe whether the net charge $Q$ and infinitesimal charge $d q$ in the three objects shown in FIG. 1 would increase, decrease, or stay the same if the objects were (dimensionally) halved while keeping the charge densities constant. We expected students to state that $d q$ will remain unchanged since the charge density remained unchanged. The results indicate that $15 \%$ of the students thought that $d q$ was entirely proportional to the overall size of the object being considered, and $14 \%$ of the students thought that $d q$ would increase when the objects were halved. Two examples of incorrect explanations were: "Increase because the charge would be distributed along a smaller area." and "Decrease, because half of the surface carrying the charge is gone." 
Questions 2,3,4 \& 5 refer to the three objects shown below. All of these objects contain the same total charge $Q$.
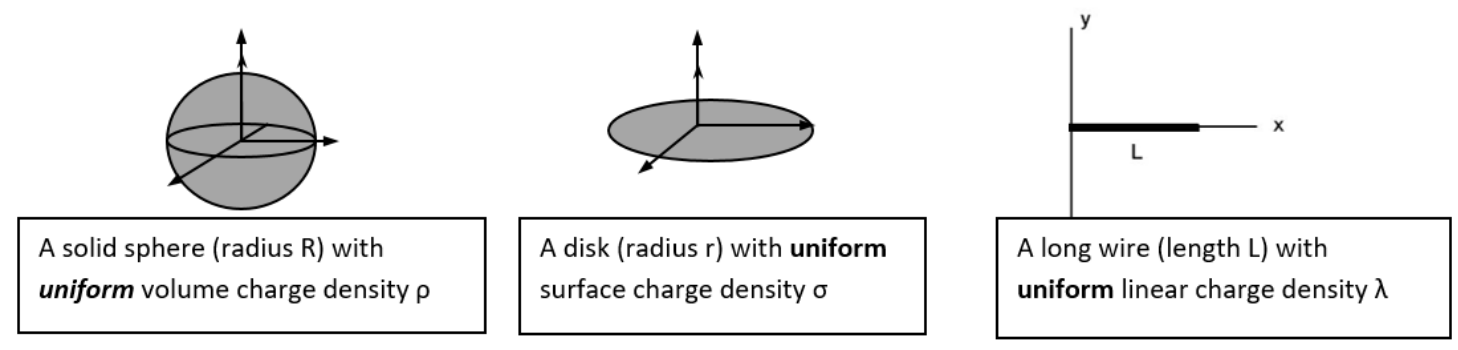

FIG. 1. Illustrations of volume, surface and line charge distributions.

TABLE II. Distributions of student responses to the questions about changes in $Q$ and $d q$ when objects were halved.

\begin{tabular}{clc}
\hline \hline Answers & $d q(\boldsymbol{\%})$ & $Q(\boldsymbol{\%})$ \\
\hline Stays Constant & $\mathbf{3 6}$ (correct) & 20 \\
Decreases & 15 & $\mathbf{3 6}$ (correct) \\
Increases & 14 & 6 \\
Unanswered & 34 & 39 \\
\hline \hline
\end{tabular}

For the part of the question related to the net charge $Q$ possessed by each object after the size reduction, we expected students to pick out the option suggesting a decrease in the total charge. Since the charge density is kept constant, a decrease in the dimensions of a charge carrying object results in a decrease in the net charged possessed by the object. Roughly half of the students who thought that the net charge $Q$ would increase argued that the charge would be more concentrated in a smaller object, whereas $20 \%$ of the students thought that the overall charge is independent of the dimensions of the object. Two examples of incorrect explanations were: "Greater because it has less area to cover." and "Equivalent because we can't have less than what we started with." The details of students' responses to both parts of this ques- tions are summarized in $T A B L E I I$.

\section{CONCLUSIONS}

This paper presents a research study aimed at understanding student difficulties with continuous charge distributions. We find that students taking introductory calculus based physics courses tend to struggle with a range of concepts that pertain to continuous charge distributions and infinitesimal charge. The students in our study had difficulty visualizing and formulating continuous charge distributions. When presented with questions involving the infinitesimal charge element, students struggled to use knowledge from their calculus classes in a physical context to arrive at the correct solution. Students were unable to perform sufficiently well in the conceptual questions, revealing issues with students' fundamental understanding of the material. Our results also hint at the disconnect between mathematical formalism and physics. Instructors teaching introductory physics courses should carefully assess their students' grasp of calculus in a physical context and provide necessary supplemental material to strengthen this skill. Relevant studies on mathematical education should be carefully considered when designing material for introductory physics courses that involve integral expressions.
[1] Galili, D. Kaplan, and Y. Lehavi, American Journal of Physics 74, 337 (2006).

[2] B. E. S. Eylon and U. Ganiel, International Journal of Science Education 12, 79 (1990).

[3] M. Saglam and R. Millar, International Journal of Science Education 28, 543 (2006).

[4] F. R. Yeatts and J. R. Hundhausen, American Journal of Physics 60, 716 (1992).
[5] N. Mahir, International Journal of Mathematical Education in Science and Technology 40, 201 (2009).

[6] A. Orton, Educational Studies in Mathematics 14, 1 (1983).

[7] S. R. Jones, The Journal of Mathematical Behavior 38,1, (2015).

[8] D.-H. Nguyen and N. S. Rebello, Physical Review Special Topics - Physics Education Research 7, (2011).

[9] C. S. Wallace and S. V. Chasteen, Physical Review Special Topics - Physics Education Research 6, (2010).

[10] B. R. Wilcox, M. D. Caballero, R. E. Pepper, and S. J. Pollock, American Institute of Physics Conference Series 1513, (2013). 